\title{
A master formulation of organic extra virgin olive oils increases HDL cholesterol and albumin serum levels in kidney patients, and ameliorates atopic dermatitis in children and adults
}

\author{
V. G. Villarrubia ${ }^{1}$, S. Vidal ${ }^{2}$, F. Borrego-Utiel ${ }^{3}$, J. M. Gil-Cunquero $^{3}$ and V. Pérez-Bañasco ${ }^{3}$ \\ ${ }^{1}$ Dpt R\&D Immunology, Bioaveda, Jaén, Spain, ${ }^{2}$ Servicio Dermatología, Hospital Gómez-Ulla, Madrid, Spain and ${ }^{3}$ Servicio \\ Nefrología, Hospital de Jaén, España
}

\begin{abstract}
Patients with chronic kidney disease (CKD), as well as patients with atopic dermatitis (AD), show severe systemic and skin immune/ inflammatory alterations. Furthermore, patients with CKD and AD exhibit disturbances of the systemic and/or the cutaneous lipid compartment, which are reflected by dyslipidaemia in CKD, and in both cases by troubles in the skin lipid composition, thus leading to dry skin. Attempting to palliate these pathogenic features, a master formulation of organic extra virgin olive oils ('oHo' ${ }^{\circledR}$, Bioaveda, Spain) was orally administered to all patients in two different pilot studies. The first trial included 19 adult patients with advanced CKD at pre-dialysis, which were compared with $12 \mathrm{CKD}$ patients having conventional/monovarietal olive oil. The second trial included 6 patients with moderate to severe $\mathrm{AD}$ associated with asthma and/or to allergic rhinitis. All patients selected for these studies were habitually consumers of conventional/monovarietal extra virgin olive oil. After one month treatment ( $20 \mathrm{ml} / \mathrm{d}$, t.i.d), only CKD patients with 'oHo' showed significant increases in HDL-cholesterol $(53-63 \mathrm{mg} / \mathrm{dl} ; P<0.01)$ and albumin $(4.1-4.5 \mathrm{~g} / \mathrm{dl} ; P<0.05)$ serum levels, and constipation diminished drastically in $>90 \%$ of cases. After one month follow-up, all parameters returned to baseline values.

Patients with AD received 'oHo' at different doses depending on age (10-60 $\mathrm{ml} / \mathrm{d})$. Eczema and pruritus disappeared in all patients after 2-3 months of the oil administration. In two cases, the 'oHo' arrest provoked the reappearance of skin lesions.

This is the first demonstration about the ability of an olive oil to enhance significantly HDL cholesterol and albumin serum levels in humans with severe inflammatory and cardiovascular risk, and to ameliorate AD in children and adults. Some intriguing immune changes will be discussed at the meeting. Larger, randomised trials are now in progress.
\end{abstract}

